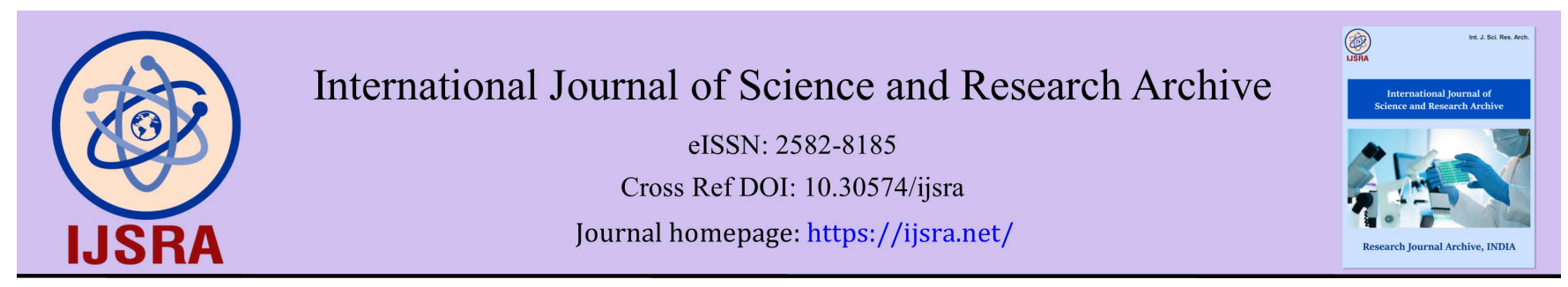

(REVIEW ARTICLE)

\title{
Cytokine storm interference
}

\section{Michael John Dochniak*}

Alleam, LLC, Minnesota, United States of America.

International Journal of Science and Research Archive, 2021, 04(01), 185-187

Publication history: Received on 10 November 2021; revised on 18 December 2021; accepted on 20 December 2021

Article DOI: https://doi.org/10.30574/ijsra.2021.4.1.0196

\begin{abstract}
Viral infections are a natural and inevitable part of life. In healthy individuals, mortality increases when the body's innate immune system quickly activates, creating a cytokine storm. A rapid and excessive release of cytokines into the bloodstream can cause acute respiratory distress syndrome and death. This communication proposes inhibiting cytokine storm development through forced atopy. Hyper-allergenic skin cream therapy stimulates adaptive immunity to support innate immunity before acute infection.
\end{abstract}

Keywords: Adaptive immunity; Atopy; Innate immunity; Influenza A; Interleukin- 6; Skin cream

\section{Introduction}

The human influenza A virus is a natural biological entity that spreads disease annually in the United States. It is the only influenza virus known to cause flu pandemics; its mutations can be highly infectious and spread quickly [1].

Historically, many deaths associated with the "Spanish Flu" pandemic of 1918-1920 may have been due to cytokine storms [2].

Most recently, evidence suggests that the severity of COVID-19 is associated with an increased level of cytokines, and other inflammatory mediators, leading to acute respiratory distress syndrome [3].

Acute respiratory distress syndrome (ARDS) arises when fluid builds up in the tiny air sacs (i.e., alveoli) in your lungs. A study indicates that ARDS is a causative syndrome of death in about 70\% of fatal COVID-19 cases [4].

David Martin, Pfizer Senior Medical Director, has stated, "A cytokine storm is generally what leads to otherwise healthy young people dying of the flu" [5]. A cytokine storm leads to the rapid proliferation and activation of $\mathrm{T}$ cells, macrophages, and natural killer cells that eventually secrete many inflammatory cytokines, chemokines, and chemical mediators [6].

The innate immune system is the first line of defense against a new pathogen (e.g., mutated virus). It also directs the qualitative and quantitative nature of adaptive immunity. During a SARS-CoV-2 infection, severe symptoms indicate an elevated expression of interleukin-6 (IL-6) [7]. IL-6 is a pleiotropic factor involved in innate and adaptive immunity [8]. Therapies designed to control a cytokine storm include blocking cytokines, corticosteroids, blood purification therapy, and stem cell therapy. Some of these therapies have entered the stage of clinical trials, adverse effects and availability have delayed their widespread application in clinical treatment $[9,10]$.

\footnotetext{
${ }^{*}$ Corresponding author: Michael John Dochniak

Alleam, LLC, Minnesota, United States of America.

Copyright (@ 2021 Author(s) retain the copyright of this article. This article is published under the terms of the Creative Commons Attribution Liscense 4.0.
} 
Hyper-allergenic skin cream therapy inhibits cytokine storm development by stimulating adaptive immunity and increasing the efficacy of cytokine pleiotropy before acute infection.

\section{Discussion}

Can hyper-allergenic skin cream therapy (i.e., forced atopy before an infection) support innate immunity to circumvent harmful inflammatory events associated with a cytokine storm? Atopy is a Type 2 immune response to environmental antigens (e.g., allergens) considered harmless. In a study published in the journal Allergy (2021) titled, "Atopic status protects from severe complications of COVID-19" the researchers concluded, "Atopic status seems to protect against the most severe, often fatal consequences of SARS-CoV2 infection. Such finding may be of help for future studies investigating how to limit the clinical consequences of this infection" [11].

In a communication published in the journal European Journal of Gastroenterology \& Hepatology (2020) titled, "Eosinophilic esophagitis: is the Th2 inflammation protective against the severe form of COVID-19?" the researchers write, "Evidence could suggest that eosinophilic esophagitis and other allergic diseases, characterized by a Th2-skewed immunity, may be protective against severe COVID-19 disease and could support the hypothesis that eosinophils have a protective effect on SARS-CoV-2 infection" [12].

In a study published in the journal Annals of Allergy, Asthma \& Immunology (2020) titled, "Atopy is predictive of a decreased need for hospitalization for coronavirus disease 2019" the researchers write, "The knowledge that atopy is associated with less severe COVID-19 outcomes can be instructive in clinical risk stratification. Further studies are needed to understand the underlying mechanism of these apparent protective physiological factors that may prove advantageous in future prevention and treatment strategies" [13].

Alternatively, does a humoral response defect contribute to cytokine storm fatality? In a study published in the journal Viruses (2019) titled, "Cytokine Storm Combined with Humoral Immune Response Defect in Fatal Hemorrhagic Fever with Renal Syndrome Case" the researchers concluded, "We propose that differences in a patient's humoral immune response to the virus could be a contributing factor in fatal HFRS. Differences in epitope recognition by antibodies from patients with different outcomes of the disease are reported regularly. It has been suggested that changes in epitope recognition could lead to inability of antibodies to interfere with virus replication. In the present HFRS case, changes in the epitope recognition were associated with a low neutralizing antibody titer, which could contribute to pathogenesis and the fatal outcome" [14].

There remains a need for immunomodulatory therapy that dampens the harmful effect of a cytokine storm. Hyperallergenic skin creams stimulate adaptive immunity to support immune system synergy. Examples of prophylactics that may affect "cytokine storm interference" are described in the United States Patent application number 20210015912 A1 (2019) titled, "Topical hyper-allergenic composition and method of treating using the same" [15].

\section{Conclusion}

Research efforts continue to explore if atopy before acute infection affects cytokine pleiotropy to inhibit cytokine storm development. In "cytokine storm interference," hyper-allergenic skin cream therapy is intended to stimulate adaptive immunity in support of innate immunity to reduce the clinical impact of a cytokine storm and minimize sequelae.

\section{Compliance with ethical standards}

\section{Acknowledgments}

The author would like to thank Denise Harmony Dunn for editing.

\section{Author disclosure}

Michael J. Dochniak is cofounder of Alleam, LLC, Minnesota, and United Sates of America. This commentary contains a discussion of an unapproved/ investigative hyper-allergenic skin cream to explore "cytokine storm interference."

\section{Author's Profile}

Michael J. Dochniak has researched and authored several books relating to Alzheimer's Disease, Artificial Intelligence, Autism Spectrum Disorders, and Climate Change through Cambridge Scholars Publishing and Nova Science Publishers. 


\section{References}

[1] CDC, Types of Influenza Viruses, Available from: https://www.cdc.gov/flu/about/viruses/types.htm

[2] Morens DM, Taubenberger JK, Harvey HA, Memoli MJ. The 1918 influenza pandemic: lessons for 2009 and the future. Crit Care Med. 2010 Apr; 38(4 Suppl): e10-20.

[3] Hojyo S, Uchida M, Tanaka K, Hasebe R, Tanaka Y, Murakami M, Hirano T. How COVID-19 induces cytokine storm with high mortality. Inflamm Regen. 1 Oct 2020; 40: 37.

[4] Huang C, Wang Y, Li X, Ren L, Zhao J, Hu Y, Zhang L, Fan G, Xu J, Gu X, Cheng Z, Yu T, Xia J, Wei Y, Wu W, Xie X, Yin W, Li H, Liu M, Xiao Y, Gao H, Guo L, Xie J, Wang G, Jiang R, Gao Z, Jin Q, Wang J, Cao B. Clinical features of patients infected with 2019 novel coronavirus in Wuhan, China. Lancet. 15 Feb 2020; 395(10223): 497-506.

[5] Breakthroughs.com, Inside a Cytokine Storm: When Your Immune System is Too Strong. Available from: https://www.breakthroughs.com/foundations-science/inside-cytokine-storm-when-your-immune-systemtoo-strong

[6] Kany S, Vollrath JT, Relja B. Cytokines in Inflammatory Disease. Int J Mol Sci. 28 Nov 2019; 20(23): 6008.

[7] Qin C, Zhou L, Hu Z, Zhang S, Yang S, Tao Y, Xie C, Ma K, Shang K, Wang W, Tian DS. Dysregulation of immune response in patients with COVID-19 in Wuhan, China. Clin. Infect. Dis. 2020.

[8] Kishimoto T. Interleukin-6: discovery of a pleiotropic cytokine. Arthritis Res Ther. 2006; 8 Suppl 2(Suppl 2): S2.

[9] Tang L, Yin Z, Hu Y, Mei H. Controlling Cytokine Storm Is Vital in COVID-19. Front Immunol. 2020 Nov 30; 11: 570993.

[10] Alijotas-Reig J, Esteve-Valverde E, Belizna C, Selva-O'Callaghan A, Pardos-Gea J, Quintana A, Mekinian A, Anunciacion-Llunell A, Miró-Mur F. Immunomodulatory therapy for the management of severe COVID-19. Beyond the anti-viral therapy: A comprehensive review. Autoimmun Rev. Jul 2020; 19(7): 102569.

[11] Scala E, Abeni D, Tedeschi A, Manzotti G, Yang B, Borrelli P, Marra A, Giani M, Sgadari A, Saltalamacchia F, Asero R. Atopic status protects from severe complications of covid-19. Allergy. mar 2021; 76(3): 899-902.

[12] Franceschini L, Macchiarelli R, Rentini S, Biviano I, Farsi A. Eosinophilic esophagitis: is the Th2 inflammation protective against the severe form of COVID-19? Eur J Gastroenterol Hepatol. Dec 2020; 32(12): 1583.

[13] Keswani A, Dhana K, Rosenthal JA, Moore D, Mahdavinia M. Atopy is predictive of a decreased need for hospitalization for coronavirus disease 2019. Ann Allergy Asthma Immunol. Oct 2020; 125(4): 479-481.

[14] Garanina E, Martynova E, Davidyuk Y, Kabwe E, Ivanov K, Titova A, Markelova M, Zhuravleva M, Cherepnev G, Shakirova VG, Khaertynova I, Tarlinton R, Rizvanov A, Khaiboullina S, Morzunov S. Cytokine Storm Combined with Humoral Immune Response Defect in Fatal Hemorrhagic Fever with Renal Syndrome Case, Tatarstan, Russia. Viruses. 2 Jul 2019; 11(7): 601.

[15] Dochniak MJ, Alleam LLC, assignee. Topical hyper-allergenic composition and method of treating using the same. United States Patent application number 20210015912 A1. 16 July 2019. Available from: https://uspto.report/patent/app/20210015912 